\title{
Tumor burden as possible biomarker of outcome in advanced NSCLC patients treated with immunotherapy: a single center, retrospective, real-world analysis
}

Edoardo Lenci $^{1}{ }^{\oplus}$, Giulia Marcantognini ${ }^{1}\left(\mathbb{C}\right.$, Valeria Cognigni ${ }^{1}$, Alessio Lupi $^{1}{ }^{\oplus}$, Silvia Rinaldi ${ }^{1}$, Luca

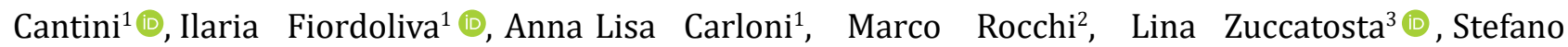
Gasparini $^{3}\left(\right.$ (D), Rossana Berardi ${ }^{*}{ }^{*}$ (1)

${ }^{1}$ Clinical Oncology, Università Politecnica delle Marche, Azienda Ospedaliero-Universitaria Ospedali Riuniti, 60126 Ancona, Italy ${ }^{2}$ Department of Biomolecular Sciences, University of Urbino Carlo Bo, 61029 Urbino, Italy

${ }^{3}$ Operative Unit of Pneumology, Ospedali Riuniti University Hospital, 60126 Ancona, Italy

*Correspondence: Rossana Berardi, Clinical Oncology, Università Politecnica delle Marche, Azienda Ospedaliero-Universitaria Ospedali Riuniti, Via Tronto 10/A, 60020 Ancona, Italy. r.berardi@univpm.it

Academic Editor: Marius Ilié, Université Côte d’Azur; Nice University Hospital, France

Received: January 19, 2021 Accepted: April 19, 2021 Published: June 28, 2021

Cite this article: Lenci E, Marcantognini G, Cognigni V, Lupi A, Rinaldi S, Cantini L, et al. Tumor burden as possible biomarker of outcome in advanced NSCLC patients treated with immunotherapy: a single center, retrospective, real-world analysis. Explor Target Antitumor Ther. 2021;2:227-39. https://doi.org/10.37349/etat.2021.00043

\section{Abstract}

Aim: The role of tumor burden (TB) for patients with non-small cell lung cancer (NSCLC) receiving immunotherapy is still unknown. The aim of this analysis was to analyze the prognostic value of TB in a realworld sample of advanced NSCLC patients.

Methods: Sixty-five consecutive patients with advanced NSCLC treated with immunotherapy as first or second line therapy were retrospectively analyzed between August 2015 and February 2018. TB was recorded at baseline considering sites and number of metastases, thoracic vs. extrathoracic disease, measurable disease (MD) vs. not-MD (NMD) and evaluating dimensional aspects as maximum lesion diameter (cut-off $=$ $6.3 \mathrm{~cm}$ ), sum of the 5 major lesions diameters (cut-off $=14.3 \mathrm{~cm}$ ), and number of sites of metastases (cut-off $>4$ ). All cut-offs were calculated by receiver operating characteristic curves. Median overall survival (OS) was estimated using Kaplan-Meier method. A Cox regression model was carried out for univariate and multivariate analyses.

Results: Median age was 70 years and most patients (86.2\%) had a good performance status (PS-Eastern Cooperative Oncology Group < 2). No significant difference in OS was noted between subgroups of patients according to TB. Bone metastases (BM) had a negative prognostic impact [median OS (mOS), 13.8 vs. 70.0 months, $P=0.0009$; median progression free survival in the second line (mPFS2) 2.97 vs. 8.63 months; $P=$ 0.0037]. Patients with NMD had a poorer prognosis (mOS, 15.9 months $v s$. not reached, $P<0.0001$; mPFS2 3.8 vs. 12.2 months; $P=0.0199$ ). Patients with disease limited to the thorax had a better prognosis compared to patients with involvement of extrathoracic sites (mOS, 70 vs. 17.3 months; $P=0.0136$ ). Having more than 4 metastatic sites resulted as a negative prognostic factor (mOS, 15.9 vs. 25.2 months; $P=0.0106$ ). At 
multivariate analysis, BM, NMD, extrathoracic disease and number of sites of metastases $>4$ were negative prognostic factors $(P<0.0001)$.

Conclusions: This study underlines the negative prognostic impact of specific metastatic sites, presence of NMD and extrathoracic disease in advanced NSCLC patients treated with immunotherapy. However, TB does not appear to affect the outcome of these patients.

\section{Keywords}

Immunotherapy, non-small cell lung cancer, tumor burden

\section{Introduction}

Lung cancer is the second most commonly diagnosed cancer in the world, recording 2,206 million new cases, corresponding to $11.4 \%$ of new diagnoses [1]. Despite the important results obtained from cancer research, it remains the leading cause of cancer-related death both in men and in women [2].

In the last few years, the advent of immune checkpoint inhibitors (ICIs) has dramatically changed the treatment landscape of advanced non-small cell lung cancer (NSCLC) patients, with a significant impact on both quality of life and overall survival (OS) [3-7]. Nowadays, standard systemic treatment for advanced non oncogene-addicted NSCLC is represented by the combination of chemotherapy and ICIs, which shown to be effective in all subgroups of patients regardless of programmed death-ligand 1 (PD-L1) expression, with the larger benefit being revealed when PD-L1 expression is upper than $50 \%[7,8]$. According to current approval in Italy, ICIs in combination with chemotherapy are used as first-line treatment for advanced NSCLC patients with PD-L1 expression lower than 50\%, while monotherapy with pembrolizumab [a fully humanized immunoglobulin G4 (IgG4) monoclonal antibody against programmed cell death-1 (PD-1)] is indicated in the same setting when PD-L1 expression is $\geq 50 \%$ [9].

Despite the success of ICIs, due to tumor heterogeneity, still not all patients derive benefit from this approach and new biomarkers are needed to improve patient selection.

Currently, PD-L1 expression is the only prospectively validated biomarker of immunotherapy response in patients with non-oncogene addicted NSCLC [10]. PS and specific hematologic values, such as neutrophilto-lymphocyte ratio, lactate dehydrogenase ( $\mathrm{LDH}$ ), and serum albumin have been recognized as promising prognostic factors both in patients treated with chemotherapy and with immunotherapy [11-15]. Furthermore, recent research has shown promise for alternative markers of response, including tumor-infiltrating immune cell $[16,17]$, expression of indoleamine 2,3-dioxygenase (ID0), microbiome profile, mutational load, mismatch repair deficiency (dMMR), expression of inflammatory genes [18] baseline metabolic tumor volume [19], body mass index [20], smoking status [21] and concomitant medications [22].

The role played by the metastatic site in prognosis prediction of NSCLC patients still represents a matter of debate. In particular, liver metastases seem to be related to a worse outcome, but the role of other metastatic sites such as bone, central nervous system (CNS), lymph nodes, and lung remains controversial [23-25].

Tumor burden (TB) is assessed as the tumor load detected with radiological exams. Albeit the definition of TB is not consistent across studies, it has been hypothesized that a high TB at diagnosis or at the start of first-line treatment may be correlated with a worse prognosis [26-28]. The prognostic value of TB has been investigated in different solid tumors, such as melanoma, colon-rectal cancer, renal cell cancer and hepatocarcinoma, yet its assessment differed in each study [26-28]. In some studies, TB was calculated as the sum of diameters of target lesions, in other cases as the number and diameter of metastases.

The aim of this study is to investigate the potential prognostic value of specific sites of metastasis in a real-world cohort of advanced NSCLC patients, treated with immunotherapy. Thus, we evaluated if TB should play a role as predictor of clinical outcome. 


\section{Materials and methods}

\section{Patient characteristics}

In our study, we retrospectively analyzed data from 65 patients with NSCLC treated with first/second-line immunotherapy, at our institution (Clinical Oncology, Azienda Ospedaliero-Universitaria Ospedali Riuniti, Ancona), between August 2015 and February 2018.

\section{Inclusion criteria included:}

1) Age > 18 years old;

2) Eastern Cooperative Oncology Group (ECOG)-PS $\leq 3$;

3) Histologically confirmed diagnosis of NSCLC;

4) Patients treated with first/second-line immunotherapy with nivolumab, atezolizumab or pembrolizumab;

5) epithelial growth factor receptor (EGFR), ROS proto-oncogene $1(R O S-1)$ and anaplastic lymphoma kinase $(A L K)$ wild type tumor.

\section{Exclusion criteria included:}

1) Patients undergoing exclusively chemotherapy and/or third or subsequent lines of immunotherapy;

2) Pre-existing diseases or condition that contraindicated immunotherapy:

- active systemic autoimmune disease;

- history of severe hypersensitivity to another monoclonal antibody;

- history of severe immune-related adverse reactions from treatment with ICIs or anti-CTLA4;

- severe active infections;

- conditions of severe immunodeficiency;

- use of systemic corticosteroids (equivalent to >10 mg of prednisone/day);

3) EGFR, ROS-1 or $A L K$ mutated tumor.

Depending on the temporal context in which this study has been conducted, our cohort included NSCLC patients who received monotherapy with ICIs as first or second line, before the results of clinical trial on chemo-immunotherapy combination were released. A flow chart of the study population is available in the supplementary material (Figure S1).

\section{Clinical variables}

Collected data included demographic characteristics (gender, age), smoking habit, comorbidities and concomitant therapies, type of surgical intervention (if performed), number and sites of lymph nodes in case of lymphadenectomy, type of adjuvant therapy (if administered), tumor histotype and grading, PD-L1 expression, presence of mutation or rearrangement of EGFR, ALK, ROS-1 genes, tumor stage, sites and number of metastases, PS-ECOG at diagnosis and at every different therapy line start, type of chemotherapy or immunotherapy, type of maintenance therapy (if given), number and type of grade 3-4 toxicities, type and site of radiotherapy (if effected), laboratory parameters including hemoglobin, absolute count of neutrophils, lymphocytes, monocytes and eosinophils, platelets, sodium, albumin, LDH and alkaline phosphatase (AP). For all previous values, the cut-offs commonly used in clinical practice were considered.

Tumor response to immunotherapy was assessed every three months from start of treatment and until progression, regardless of the type of ICI (pembrolizumab, atezolizumab, nivolumab) used. All patients underwent a computed tomography (CT)-scan before the start of treatment and then every three months until clinical or radiological progression or death. Tumor response to immunotherapy was assessed by an expert radiologist using IgG4 immune Response Evaluation Criteria in Solid Tumors (iRECIST) criteria and according to approved guidelines [29]. Fluorodeoxyglucose (FDG)-positron emission tomography (PET)/CT evaluation has been performed only in case of lesions suspected for metastases at CT-scan. 
As a universally accepted method still must be found, we decided, according to literature, to determine TB considering:

1) the number and the size of metastases (according to work by Sasaki, et al. [28], TB was defined as the number and size of metastases);

2) the diameters of the main target lesion (according to work by lacovelli, et al. [26], TB was defined as the major diameters of the target lesions);

3 ) the total diameters of the 5 biggest target lesions (according to work by Katsurada, et al. [30], TB defined as the sum of the major diameters of the target lesions).

We have evaluated different definitions of TB in a cohort of NSCLC patients treated with immunotherapy.

All the previous parameters were calculated by looking at the baseline radiological assessment.

We evaluated those dimensional aspects calculating the following cut-offs with receiver operating characteristic (ROC) curve: maximum lesion diameter (cut-off $=6.3 \mathrm{~cm}$ ); sum of the 5 major lesions diameters (cut-off $=14.3 \mathrm{~cm}$ ); number of sites of metastases (cut-off $>4$ ).

We considered as "not measurable" the following lesions: ascites, pleural pouring, pericardial effusion, lung lymphangitis, bone metastases (BM), leptomeningeal metastasis.

We compared survival differences between patients with exclusively thoracic vs. extra thoracic disease and with measurable vs. not-measurable tumor lesions.

Finally, we compared survival differences between patients with 4 or more sites of metastasis vs. those with 3 or less sites of metastasis.

\section{Statistical analysis}

OS was defined as the time interval between the date of first cycle of ICIs administration and death; for patients who had not died, OS was censored at time of last follow-up. PFS2 was defined as the time interval between start of ICIs therapy and tumor progression or death; for patients who had not experienced tumor progression, PFS2 was censored at time of last follow-up. We evaluated PFS2 due to the small number of patients treated with first-line immunotherapy; these patients were excluded from the PFS2 analysis. Survival distribution was estimated using Kaplan-Meier curves and survival differences were evaluated using the logrank test. Variables that achieved statistical significance $(P<0.05)$ at univariate analysis were included in multivariate analysis using multiple Cox regression to identify independent prognostic factors by calculating their hazard ratios and their 95\% confidence intervals (95\% CI) [31]. Statistical analysis was conducted using MedCalc software version 19.1 for Windows.

\section{Results}

\section{Patients' characteristics}

Sixty-five NSCLC patients were retrospectively included in this study.

In the whole cohort, median age at diagnosis was 70 years (range $25-86$ years) and 48 (73.8\%) of patients were men. Almost all of the patients (93.8\%) were current or former smokers. At the start of immunotherapy, $82.6 \%$ of patients in our cohort had PS-ECOG lower than 2.

In $56.9 \%$ of cases, histologic subtype was represented by non-squamous carcinoma. Forty-four (66.7\%) patients showed not-measurable lesions with or without measurable disease and twenty-one patients (32.3\%) showed only measurable disease at the time of diagnosis. Thirty-three patients (50.8\%) had at least one extrathoracic localization of disease. Ten patients received immunotherapy in first line (Table 1).

At a median follow up of 21 months, mOS was 23.3 months while median PFS to immunotherapy in the second line (mPFS2) was 5.3 months.

The median PFS to immunotherapy in the first line was not considered due to the small number of patients. 
Table 1. Patients' clinical characteristics

\begin{tabular}{|c|c|c|c|}
\hline Clinical characteristics & & Patients $n$ & $\%$ \\
\hline \multirow[t]{2}{*}{ Sex } & Male & 48 & 73.8 \\
\hline & Female & 17 & 26.2 \\
\hline \multirow[t]{2}{*}{ Histology } & Squamous & 28 & 43.1 \\
\hline & Non-squamous & 37 & 56.9 \\
\hline \multirow[t]{2}{*}{ Age at diagnosis } & $<70$ years & 35 & 53.8 \\
\hline & $\geq 70$ years & 30 & 46.2 \\
\hline \multirow[t]{3}{*}{ Smoking } & Smokers & 16 & 24.6 \\
\hline & Non-smokers & 4 & 6.2 \\
\hline & Former smokers & 45 & 69.2 \\
\hline \multirow[t]{2}{*}{ PS-ECOG (at start of immunotherapy) } & $\geq 2$ & 9 & 13.8 \\
\hline & $<2$ & 56 & 86.2 \\
\hline \multirow[t]{7}{*}{ Metastases sites } & Bone & 28 & 43.1 \\
\hline & Pulmonary & 47 & 72.3 \\
\hline & Adrenal & 15 & 23.1 \\
\hline & Lymph nodes & 51 & 78.5 \\
\hline & Pleural & 22 & 33.8 \\
\hline & Cerebral & 8 & 12.3 \\
\hline & Liver & 9 & 13.8 \\
\hline \multirow[t]{4}{*}{ Disease characteristics } & Measurable & 21 & 32.3 \\
\hline & Not measurable & 44 & 67.7 \\
\hline & Extra-thoracic localization of disease & 33 & 50.8 \\
\hline & Thoracic localization of disease & 32 & 49.2 \\
\hline \multirow[t]{2}{*}{ Number of sites of metastases } & $<4$ & 48 & 73.8 \\
\hline & $\geq 4$ & 17 & 26.2 \\
\hline \multirow[t]{2}{*}{ Immunotherapy } & First line & 10 & 15.4 \\
\hline & Second line & 55 & 84.6 \\
\hline mOS & 23.3 months & & \\
\hline mPFS2 & 5.3 months & & \\
\hline
\end{tabular}

\section{Association between clinical characteristics and $0 S$}

In the whole cohort of patients, no difference in clinical outcome was observed considering sex, age (considering 70 years as cut-off [14, 32]) and smoking status.

Looking at TB, the cut-off calculated with the ROC curve was $6.3 \mathrm{~cm}$ for the largest tumoral lesion's diameter and $14.3 \mathrm{~cm}$ for the sum of the 5 largest lesion's diameters. No significant difference in OS was noted between subgroups of patients according to TB.

Patients with $\geq 4$ metastatic sites showed a worse survival when compared with patients with $<4$ metastatic sites [mOS, 15.9 vs. 25.2 months; Hazard Ratio (HR): 2.56, 95\% CI 1.33-8.63; $P=0.0106$; Figure 1].

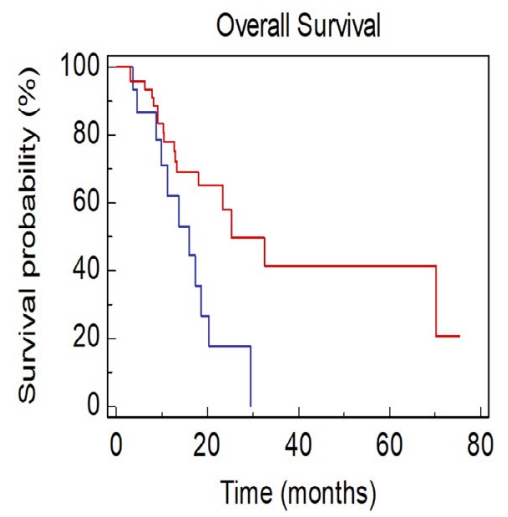

Figure 1. OS of NSCLC patients stratified by number of sites of metastases BLUE $\geq 4$ vs. RED $<4$ ) 
Patients with a poor PS (PS-ECOG $\geq 2$ ) showed a significantly lower OS compared to those with PS-ECOG 0-1 (mOS, 9.1 vs. 24.0 months, HR: 2.43, 95\% CI 1.19-10.56; $P=0.0226$; Figure 2).

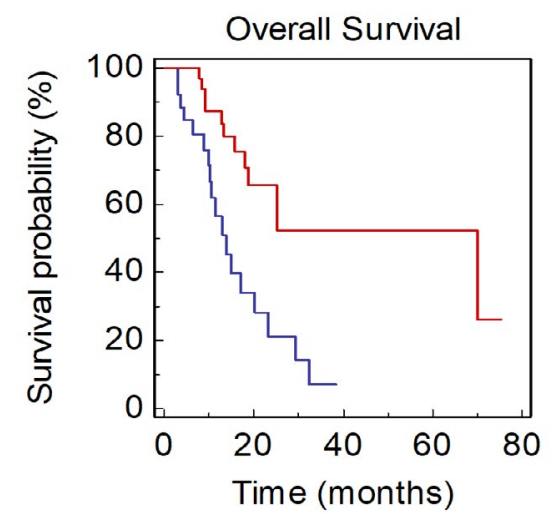

Figure 2. OS of NSCLC patients stratified by PS (BLUE: ECOG $\geq 2$ vs. RED: ECOG: 0-1)

Presence of liver, pulmonary, lymph nodes, pleural, adrenal and cerebral metastases did not result significantly associated with OS.

Looking at metastatic sites at baseline, presence of BM was associated with a significantly worse OS (mOS, 13.8 vs. 70.0 months, HR: 3.27, 95\% CI 1.73-8.28; $P=0.0009$; Figure 3).

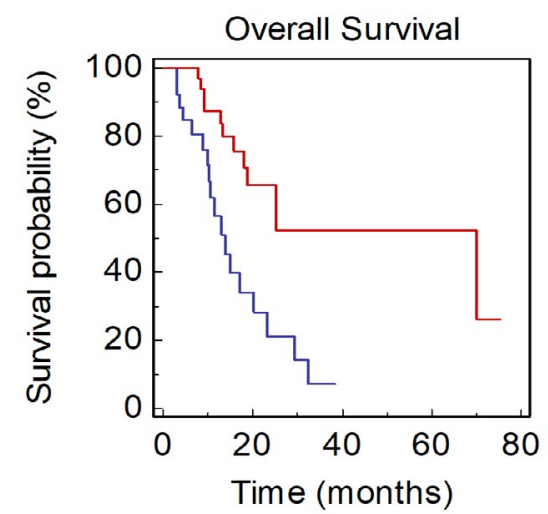

Figure 3. OS of NSCLC patients stratified by BM (BLUE: present vs. RED: absent)

Patients with not-measurable disease at baseline had a median OS of 15.9 months, while mOS was not reached in patients with only measurable disease (HR: 0.09, 95\% CI 0.08-0.44; $P<0.0001$; Figure 4).

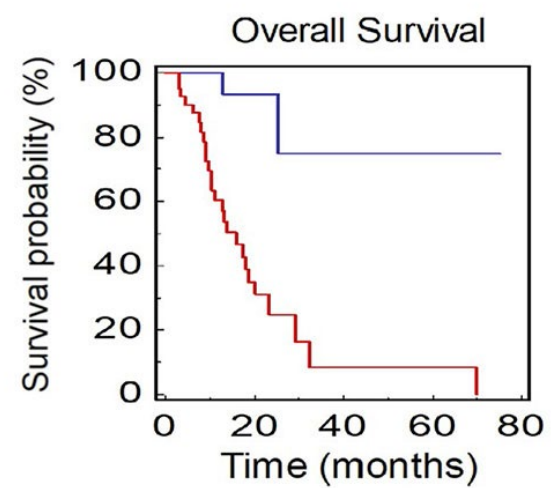

Figure 4. OS of NSCLC patients stratified by presence of measurable or not-measurable disease (BLUE: measurable vs. RED: not-measurable)

Patients with only thoracic disease had a better prognosis compared to patients with extrathoracic thoracic disease (mOS, 70.0 vs. 17.3 months, HR: 0.40, 95\% CI 0.17-0.82; $P=0.0136$; Figure 5). 


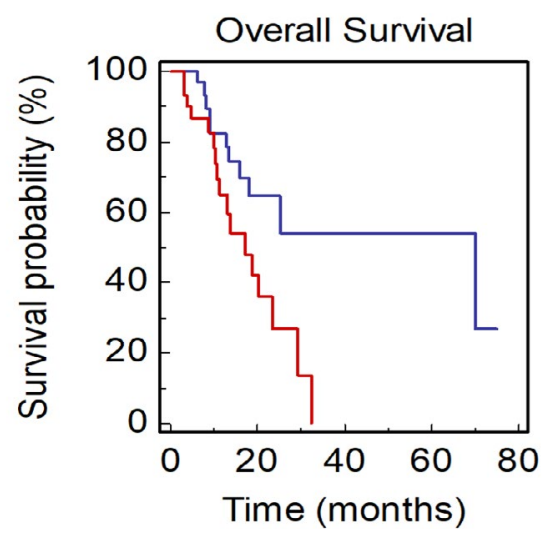

Figure 5. OS of NSCLC patients stratified by site of metastasis (BLUE: thoracic vs. RED: extrathoracic)

The multivariate analysis confirmed BM, non-measurable disease, extrathoracic disease and a number of sites of metastases $>4$ as negative independent prognostic factors $(P<0.0001)$ (Table 2$)$.

Table 2. Multivariable analyses for OS

\begin{tabular}{|c|c|c|c|c|}
\hline \multirow[t]{2}{*}{ Clinical parameter } & \multicolumn{2}{|c|}{ Univariate cox regression } & \multicolumn{2}{|c|}{ Multivariate cox regression } \\
\hline & $\mathrm{HR}(95 \% \mathrm{CI})$ & $P$-value & $\begin{array}{l}\text { Exponentiation of the } \\
\mathrm{B} \text { coefficient (EXP B, } \\
95 \% \mathrm{Cl} \text { ) }\end{array}$ & $P$-value \\
\hline Gender (male vs. female) & $0.72(0.30-1.64)$ & 0.4115 & & \\
\hline PS-ECOG ( $\geq 2$ vs. $<2)$ & $2,43(1,19-10.56)$ & 0.0226 & $0.37(0.12-1.14)$ & 0.0840 \\
\hline Age $(\geq 70$ vs. $<70)$ & $1.44(0.70-2.98)$ & 0.3195 & & \\
\hline Histotype (squamous vs. non-squamous) & $1.08(0.53-2.21)$ & 0.8328 & & \\
\hline Smoke (yes vs. no) & $0.38(0.17-3.95)$ & 0.8074 & & \\
\hline BM (no vs. yes) & $3.28(1.73-8,28)$ & 0.0009 & $0.19(0.05-0.78)$ & 0.0222 \\
\hline Pulmonary metastases (no vs. yes) & $1.08(0.36-3.23)$ & 0.8845 & & \\
\hline Liver metastases (no vs. yes) & $0.45(0.11-1.20)$ & 0.0984 & & \\
\hline Cerebral metastases (no vs. yes) & $0.78(0.20-2.85)$ & 0.6751 & & \\
\hline Adrenal metastases (no vs. yes) & $0.66(0.22-1.62)$ & 0.3124 & & \\
\hline Lymph nodes metastases (no vs. yes) & $1.29(0.53-3.33)$ & 0.5502 & & \\
\hline Pleural metastases (no vs. yes) & $0.58(0.24-1.21)$ & 0.1368 & & \\
\hline Maximum lesion diameter (cut-off $=6.3 \mathrm{~cm}$ ) & $0.80(0.35-1.86)$ & 0.6097 & & \\
\hline $\begin{array}{l}\text { Sum of the } 5 \text { major lesions diameters (cut-off }= \\
14.3 \mathrm{~cm} \text { ) }\end{array}$ & $1.35(0.46-4.31)$ & 0.5438 & & \\
\hline Intrathoracic vs. extrathoracic disease & $0.40(0.17-0.82)$ & 0.0136 & $0.12(0.03-0.52)$ & 0.0212 \\
\hline Measurable vs. not-measurable disease & $0.09(0.08-0.44)$ & $<0.0001$ & $0.14(0.02-0.74$ & 0.0216 \\
\hline Number of metastatic sites $(\geq 4$ vs. $<4$ ) & $2.56(1.33-8.63)$ & 0.0106 & $0.65(0.26-0.87)$ & 0.0372 \\
\hline
\end{tabular}

\section{Association between clinical characteristics and PFS2}

Considering the 55 patients receiving immunotherapy as second line treatment, male had a longer PFS2 (mPFS2, 5.83 vs. 3.93 months, HR: 0.44, 95\% CI 0.13-0.87; $P=0.0248$ ).

No difference in clinical outcome was observed considering sex, age (cut-off: 70 years), PS and smoking status.

No significant difference in PFS2 was noted between subgroups of patients according to the largest tumoral lesion's diameter and for the sum of the 5 largest lesion's diameters, intrathoracic localization and number of metastatic sites.

Patients with at least one bone metastasis showed a significantly lower PFS2 compared to those without BM (mPFS2, 2.97 vs. 8.63 months, HR: 2.60, 95\% CI 1.47-7.23; $P=0.0037$; Figure 6).

Presence of liver, pulmonary, lymph nodes, pleural, adrenal and cerebral metastases did not result significantly associated with PFS2. 


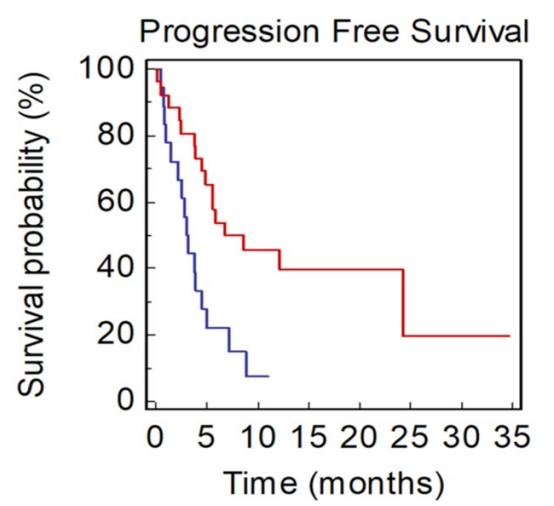

Figure 6. PFS2 of NSCLC patients stratified by BM (BLUE: present vs. RED: absent)

Patients with not-measurable disease at baseline had a median PFS2 of 3.8 months compared to 12.2 months in patients with only measurable disease (HR: 0.40, 95\% CI 0.21-0.87; $P=0.0199$; Figure 7).

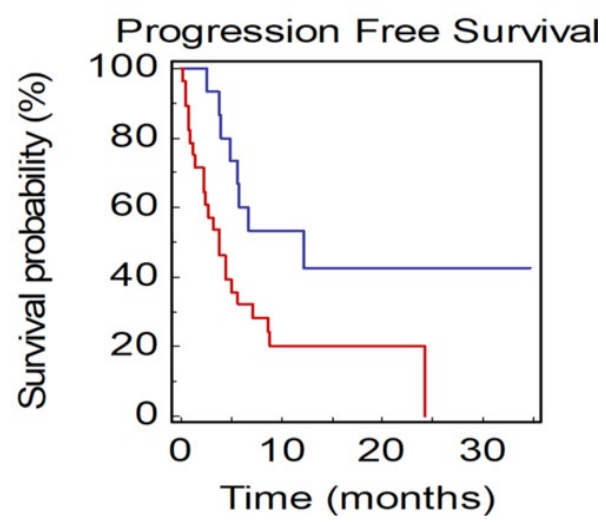

Figure 7. PFS2 of NSCLC patients stratified by presence of measurable or not-measurable disease (BLUE: measurable vs. RED: not- measurable)

The multivariate analysis confirmed female sex and BM as negative independent prognostic factors $(P=$ 0.0016; Table 3).

Table 3. Multivariable analyses for PFS2

\begin{tabular}{|c|c|c|c|c|}
\hline \multirow[t]{2}{*}{ Clinical parameter } & \multicolumn{2}{|c|}{ Univariate cox regression } & \multicolumn{2}{|c|}{ Multivariate cox regression } \\
\hline & $\mathrm{HR}(95 \% \mathrm{Cl})$ & $P$-value & $\operatorname{EXP}(B, 95 \% \mathrm{Cl})$ & $P$-value \\
\hline Gender (male vs. female) & $0.44(0.13-0.87)$ & 0.0248 & $2.56(1.08-6.07)$ & 0.0340 \\
\hline PS-ECOG ( $\geq 2$ vs. $<2)$ & $1.57(0.60-5.06)$ & 0.3039 & & \\
\hline Age $(\geq 70$ vs. $<70)$ & $1.80(0.87-3.54)$ & 0.1157 & & \\
\hline Histotype (squamous vs. non-squamous) & $1.56(0.79-3.29)$ & 0.1938 & & \\
\hline Smoke (yes vs. no) & $0.95(0.22-4.14)$ & 0.9545 & & \\
\hline BM (no vs. yes) & $2.60(1.47-7.23)$ & 0.0037 & $0.38(0.15-0.42)$ & 0.0340 \\
\hline Pulmonary metastases (no vs. yes) & $1.71(0.29-1.87)$ & 0.5209 & & \\
\hline Liver metastases (no vs. yes) & $0.55(0.15-1.41)$ & 0.1756 & & \\
\hline Cerebral metastases (no vs. yes) & $0.50(0.09-1.55)$ & 0.1769 & & \\
\hline Adrenal metastases (no vs. yes) & $1.52(0.62-3.40)$ & 0.3830 & & \\
\hline Lymph nodes metastases (no vs. yes) & $1.00(0.43-2.33)$ & 0.9996 & & \\
\hline Pleural metastases (no vs. yes) & $0.85(0.41-1.77)$ & 0.6585 & & \\
\hline Maximum lesion diameter (cut-off $=6.3 \mathrm{~cm}$ ) & $0.74(0.34-1.64)$ & 0.4687 & & \\
\hline $\begin{array}{l}\text { Sum of the } 5 \text { major lesions diameters (cut-off } \\
=14.3 \mathrm{~cm} \text { ) }\end{array}$ & $0.66(0.29-1.66)$ & 0.4071 & & \\
\hline Intrathoracic vs. extrathoracic disease & $0.71(0.33-1.46)$ & 0.3333 & & \\
\hline Measurable vs. not-measurable disease & $0.40(0.21-0.87)$ & 0.0199 & $0.58(0.22-1.54)$ & 0.2808 \\
\hline Number of metastatic sites $(\geq 4 \mathrm{vs} .<4)$ & $0.40(0.20-0.97)$ & 0.4231 & & \\
\hline
\end{tabular}




\section{Discussion}

Identifying prognostic and predictive biomarkers and designing rational combination therapy has become crucial in NSCLC patients treated with ICIs.

The standard approach to check responsiveness to ICI therapy in these patients is to detect the expression levels of PD-L1 in tumor tissues but investigating PD-L1 expression has some limitations. Specifically, around $40-60 \%$ of patients will not benefit from immunotherapy [18] and a proportion of patients between $4 \%$ and $29 \%$ will experience hyperprogression [32].

Research is moving toward the detection of reliable tools for predicting treatment efficacy and some studies have evaluated possible biomarkers both on tumor tissue (such as IDO, dMMR, tumor-infiltrating immune cells, mutational load and natural killer cells) and on peripheral blood (such as neutrophil-tolymphocyte ratio) [16-19]. Nevertheless, these parameters are not always readily available in clinical practice, thus identifying possible useful and easy-to-access predictors of immunotherapy response remains crucial.

The term TB refers to the total mass of tumor tissue carried by a patient with a malignancy, although a universal definition still does not exist.

Previous clinical evidence has shown that low baseline TB is associated with better patients' outcome in several cancers. For example, in colorectal cancer patients with resected liver metastases, TB was defined as the number and size of metastases. Patients with high TB (cut-off $\geq 8$ metastases and/or $5 \mathrm{~cm}$ ) have a worse prognosis in terms of disease-free survival (DFS) and OS (HR 1.303; CI: 1.084-1.568; $P<0.001$ ) [33]. Furthermore, in renal cancer TB has been previously defined as the sum of the major diameters of the target lesions and has a prognostic role for the response to antiangiogenic drugs such as sunitinib or bevacizumab: specifically, for a $1 \mathrm{~cm}$ increase in TB the risk of disease progression rises by 5\% [26].

Besides, in a post-hoc analysis of KEYNOTE-001 (involving advanced melanoma patients treated with pembrolizumab), a low TB was associated with a better clinical response and a better OS in the univariate analysis. The multivariate analysis confirmed it as an independent predictor of response to pembrolizumab. In addition, the complete response (CR) rate was higher in patients with lower TB, especially when associated with positive PD-L1 expression (CR rate was $37.6 \%$ in patients with low TB, $17.8 \%$ with medium TB and $4.7 \%$ with high TB) [34].

In our analysis we attempted to "quantify" TB by considering the largest diameter of the largest lesion (cut off: $6.3 \mathrm{~cm}$ ) and the sum of the largest diameters of the 5 major measurable lesions (cut off: $14.3 \mathrm{~cm}$ ). Unlike previous studies, stratification of patients in our cohort by these parameters did not reveal significant differences in terms of OS and PFS2. In an analysis from the Kobe Universital Hospital [30] which evaluated 58 NSCLC patients treated with immunotherapy from 2015 to 2018, baseline tumor size (BTS) was defined as the sum of the major diameters of the target lesions and the minor diameters of the metastatic lymph nodes (small BTS: below $101 \mathrm{~mm}$; large BTS: above $101 \mathrm{~mm}$ ). PFS and OS of patients with a large BTS was significantly shorter than that of patients with a small BTS (mPFS, large vs. small: 2.07 vs. 6.39 months; $P=$ 0.44; mOS, large vs. small: 5.85 vs. 22.28 months; $P<0.01$ ).

Regarding sites of metastases, in our study non-measurable disease and extrathoracic disease were negative independent prognostic factors. Conversely, we found that patients with only measurable disease had a better outcome in terms of PFS2 (mPFS2, not measurable vs. measurable: 3.8 months $v s$. not reached; $P=0.0199$ ). Other studies comparing these two parameters (not measurable $v s$. measurable and extrathoracic $v s$. thoracic disease) in NSCLC patients treated with ICIs are not available in literature.

Interestingly, we found that only bone localization showed an independent negative prognostic value (mPFS2, bone positive vs. bone negative: $2.97 v s .8 .63$ months; $P=0.0037$ ). This result is consistent with the previous pre-clinical and clinical data.

On one hand, preclinical studies have shown how the bone microenvironment reduces the activity of CD4 ${ }^{+}$ and $\mathrm{CD}^{+} \mathrm{T}$ cells [35]. On the other hand, in a recent retrospective study, Kawachi, et al. [36] observed that skeleton sites were not significantly associated with a shorter PFS (HR: 1.00, 95\% CI: 0.65-1.52; $P=0.986$ ) in NSCLC patients who underwent first-line pembrolizumab. In another study Tamiya et al. [37] examined 
metastatic sites of NSCLC patients treated with nivolumab and did not find a statistically significant difference in terms of PFS according to the presence of BM. In addition, they demonstrated a worse response in patients with intrapulmonary (mPFS intrapulmonary positive vs. intrapulmonary negative: 2.27 vs. 3.52 months; $P<$ 0.01 ) or liver (mPFS liver positive vs. liver negative: $3.25 v s .1 .15$ months; $P<0.001$ ) metastases.

Remarkably, in the aforementioned study the presence of 3 or more metastatic sites significantly decreased PFS (mPFS, sites $<3$ vs. sites $\geq 3: 3.67 v s$. 1.87 months; $P=0.002$ ), while in our analysis only patients with 4 or more metastatic sites were associated with worse OS (mOS, sites $<4$ vs. sites $\geq 4: 25.2$ vs. 15.9 months; $P=0.0106$ ). Noteworthy, a post-hoc analysis of Check-Mate 057 revealed that patients who progressed to nivolumab within the first 3 months had a high TB defined as more than 5 metastatic sites including liver and bone sites [38].

There are some limitations in our analysis. First, we conducted a retrospective analysis. Second, the small sample size (particularly in first line setting) hampers definitive conclusions. Third, the lack of centralization of radiological imaging might have introduced a detection bias. Finally, our population is homogeneous for some characteristics but not for others. In particular, it is unbalanced by gender (male vs. female; 73.2\% vs. $26.8 \%$, respectively) and by line of treatment (first-line $v$ s. second-line; $15.4 \% v s .84 .6 \%$, respectively).

In conclusion, immunotherapy has improved cancer patient outcomes worldwide, but most patients still do not achieve durable disease control. Therefore, the selection of patients more likely to benefit from the treatment is crucial. Our study emphasized the negative prognostic value of a poor PS and of the number of metastases, although it did not show a significant impact of BTS on patient outcome.

The role of specific metastatic sites, the presence of non-measurable disease and extrathoracic diseases represent negative prognostic factors, although our case series was too small to draw conclusions.

Large and prospective studies are needed to clarify the usefulness of TB in evaluating the outcomes of NSCLC patients undergoing immunotherapy.

\section{Abbreviations}

ALK: anaplastic lymphoma kinase

BM: bone metastases

BTS: baseline tumor size

CI: confidence interval

ECOG: Eastern Cooperative Oncology Group

EGFR: epithelial growth factor receptor

HR: hazard ratio

ICIs: immune checkpoint inhibitors

mOS: median overall survival

mPFS: median progression free survival

mPFS2: median progression free survival in second line

NMD: not-measurable disease

NSCLC: non-small cell lung cancer

OS: overall survival

PD-L1: programmed death-ligand 1

PFS2: progression free survival in second line

PS-ECOG: performance status-Eastern Cooperative Oncology Group

ROS-1: ROS proto-oncogene 1

TB: tumor burden 


\section{Supplementary materials}

The supplementary materials for this article are available at: https://www.explorationpub.com/uploads/ Article/file/100243_sup_1.pdf.

\section{Declarations}

Author contributions

EL, SR, RB and AL contributed conception and design of the study; AL, GM, VC, IF, LZ and SG organized the data; EL, SR, LC and MR performed the statistical analysis; EL, VC and AL wrote the first draft of the manuscript. All authors contributed to manuscript revision, read and approved the submitted version.

\section{Conflicts of interest}

All authors declare that there are no conflicts of interest.

\section{Ethical approval}

The study was approved by the local Ethics Committee (ID of the study 2019-146). All procedures were performed by the ethics standards of our institutional research committee and with those of the 1964 Helsinki Declaration and its later amendments or comparable ethical standards.

\section{Consent to participate}

Written informed consent was obtained from all subjects before the study.

\section{Consent to publication}

Not applicable.

\section{Availability of data and materials}

Not applicable.

\section{Funding}

Not applicable.

\section{Copyright}

(c) The Author(s) 2021.

\section{References}

1. Sung H, Ferlay J, Siegel RL, Laversanne M, Soerjomataram I, Jemal A, et al. Global cancer statistics 2020: GLOBOCAN estimates of incidence and mortality worldwide for 36 cancers in 185 countries. CA Cancer J Clin. 2021;71:209-49.

2. Jemal A, Bray F, Center MM, Ferlay J, Ward E, Forman D. Global cancer statistics. CA Cancer J Clin. 2011;61:69-90.

3. Brahmer J, Reckamp KL, Baas P, Crinò L, Eberhardt WEE, Poddubskaya E, et al. Nivolumab versus docetaxel in advanced squamous-cell non-small-cell lung cancer. N Engl J Med. 2015;373:123-35.

4. Borghaei H, Paz-Ares L, Horn L, Spigel DR, Steins M, Ready NE, et al. Nivolumab versus docetaxel in advanced nonsquamous non-small-cell lung cancer. N Engl J Med. 2015;373:1627-39.

5. Herbst RS, Baas P, Kim DW, Felip E, Pérez-Gracia JL, Han JY, et al. Pembrolizumab versus docetaxel for previously treated, PD-L1-positive, advanced non-small-cell lung cancer (KEYNOTE-010): a randomised controlled trial. Lancet. 2016;387:1540-50.

6. Rittmeyer A, Barlesi F, Waterkamp D, Park K, Ciardiello F, von Pawel J, et al. Atezolizumab versus docetaxel in patients with previously treated non-small-cell lung cancer (OAK): a phase 3, open-label, multicentre randomised controlled trial. Lancet. 2017;389:255-65. 
7. Gandhi L, Rodríguez-Abreu D, Gadgeel S, Esteban E, Felip E, De Angelis F, et al. Pembrolizumab plus chemotherapy in metastatic non-small-cell lung cancer. N Engl J Med. 2018;22:2078-92.

8. Paz-Ares L, Vicente D, Tafreshi A, Robinson A, Soto Parra H, Mazières J, et al. A randomized, placebocontrolled trial of pembrolizumab plus chemotherapy in patients with metastatic squamous NSCLC: protocol-specified final analysis of KEYNOTE-407. J Thorac Oncol. 2020;15:1657-69.

9. Associazione Italiana di Oncologia Medica [Internet]. Milano: The Association; [cited 2021 Mar 16] . Available from: https://www.aiom.it/linee-guida-aiom/

10. Mok TS, Wu YL, Kudaba I, Kowalski DM, Cho BC, Turna HZ, et al. Final analysis of the phase III KEYNOTE-042 study: pembrolizumab (Pembro) versus platinum-based chemotherapy (Chemo) as first-line therapy for patients (Pts) with PD-L1-positive locally advanced/metastatic NSCLC. Ann Oncol. 2019;30:i38.

11. Dall'Olio FG, Maggio I, Massucci M, Mollica V, Fragomeno B, Ardizzoni A. ECOG performance status $\geq 2$ as a prognostic factor in patients with advanced non small cell lung cancer treated with immune checkpoint inhibitors-a systematic review and meta-analysis of real world data. Lung Cancer. 2020;145:95-104.

12. Diem S, Schmid S, Krapf M, Flatz L, Born D, Jochum W, et al. Neutrophil-to-lymphocyte ratio (NLR) and platelet-to-lymphocyte ratio (PLR) as prognostic markers in patients with non-small cell lung cancer (NSCLC) treated with nivolumab. Lung Cancer. 2017;111:176-81.

13. Xie X, Liu J, Yang H, Chen H, Zhou S, Lin H, et al. Prognostic value of baseline neutrophil-to-lymphocyte ratio in outcome of immune checkpoint inhibitors. Cancer Invest. 2019;37:265-74.

14. Lenci E, Cantini L, Pecci F, Cognigni V, Agostinelli V, Mentrasti G, et al. The gustave roussy immune (GRIm)-score variation is an early-on-treatment biomarker of outcome in advanced non-small cell lung cancer (NSCLC) patients treated with first-line pembrolizumab. J Clin Med. 2021;10:1005.

15. Cortellini A, Tiseo M, Banna GL, Cappuzzo F, Aerts JGJV, Barbieri F, et al. Clinicopathologic correlates of first-line pembrolizumab effectiveness in patients with advanced NSCLC and a PD-L1 expression of $\geq$ 50\%. Cancer Immunol Immunother. 2020;69:2209-21.

16. Cózar B, Greppi M, Carpentier S, Narni-Mancinelli E, Chiossone L, Vivier E. Tumor-infiltrating natural killer cells. Cancer Discov. 2021;11:34-44.

17. Li XD, Ji M, Zheng X, Ning ZH, Wu J, Lu B, et al. Evaluation of tumor response to cytokine-induced killer cells therapy in malignant solid tumors. J Transl Med. 2014;12:215.

18. Maleki Vareki S, Garrigós C, Duran I. Biomarkers of response to PD-1/PD-L1 inhibition. Crit Rev Oncol/ Hematol. 2017;116:116-24.

19. Chardin D, Paquet M, Schiappa R, Darcourt J, Bailleux C, Poudenx M, et al. Baseline metabolic tumor volume as a strong predictive and prognostic biomarker in patients with non-small cell lung cancer treated with PD1 inhibitors: a prospective study. J Immunother cancer. 2020;8:e000645.

20. Cortellini A, Ricciuti B, Tiseo M, Bria E, Banna GL, Aerts JGJV, et al. Baseline BMI and BMI variation during first line pembrolizumab in NSCLC patients with a PD-L1 expression $\geq 50 \%$ : a multicenter study with external validation. J Immunother Cancer. 2020;8:e001403.

21. Cortellini A, De Giglio A, Cannita K, Cortinovis DL, Cornelissen R, Baldesarri C, et al. Smoking status during first-line immunotherapy and chemotherapy in NSCLC patients: a case-control matched analysis from a large multicenter study. Thorac Cancer. 2021;12:880-9.

22. Cantini L, Pecci F, Hurkmans DP, Belderbos RA, Lanese A, Copparoni C, et al. High-intensity statins are associated with improved clinical activity of PD-1 inhibitors in malignant pleural mesothelioma and advanced non-small cell lung cancer patients. Eur J Cancer. 2021;144:41-8.

23. Riihimäki M, Hemminki A, Fallah M, Thomsen H, Sundquist K, Sundquist J, et al. Metastatic sites and survival in lung cancer. Lung Cancer. 2014;86:78-84. 
24. Badawy AA, Khedr G, Omar A, Bae S, Arafat W, Grant S. Site of metastases as prognostic factors in unselected population of stage IV non-small cell lung cancer. Asian Pacific J Cancer Prev. 2018;19: 1907-10.

25. Li J, Zhu H, Sun L, Xu W, Wang X. Prognostic value of site-specific metastases in lung cancer: a population based study. J Cancer. 2019;10:3079-86.

26. Iacovelli R, Lanoy E, Albiges L, Escudier B. Tumour burden is an independent prognostic factor in metastatic renal cell carcinoma. BJU Int. 2012;110:1747-53.

27. Seban RD, Mezquita L, Berenbaum A, Dercle L, Botticella A, Le Pechoux C, et al. Baseline metabolic tumor burden on FDG PET/CT scans predicts outcome in advanced NSCLC patients treated with immune checkpoint inhibitors. Eur J Nucl Med Mol Imaging. 2020;47:1147-57.

28. Sasaki K, Morioka D, Conci S, Margonis GA, Sawada Y, Ruzzenente A, et al. The tumor burden score: a new "metro-ticket" prognostic tool for colorectal liver metastases based on tumor size and number of tumors. Ann Surg. 2018;267:132-141.

29. Seymour L, Bogaerts J, Perrone A, Ford R, Schwartz LH, Mandrekar S, et al. iRECIST: guidelines for response criteria for use in trials testing immunotherapeutics. Lancet Oncol. 2017;18:e143-52.

30. Katsurada M, Nagano T, Tachihara M, Kiriu T, Furukawa K, Koyama K, et al. Baseline tumor size as a predictive and prognostic factor of immune checkpoint inhibitor therapy for non-small cell lung cancer. Anticancer Res. 2019;39:815-25.

31. Fagerland MW, Hosmer DW, Bofin AM. Multinomial goodness-of-fit tests for logistic regression models. Stat Med. 2008;27:4238-53.

32. Frelaut M, Le Tourneau C, Borcoman E. Hyperprogression under immunotherapy. Int J Mol Sci. 2019;20:2674.

33. Gomez D, Morris-Stiff G, Toogood GJ, Lodge JPA, Prasad KR. Interaction of tumour biology and tumour burden in determining outcome after hepatic resection for colorectal metastases. HPB. 2010;12:84-93.

34. Ribas A, Hamid O, Daud A, Hodi FS, Wolchok JD, Kefford R, et al. Association of pembrolizumab with tumor response and survival among patients with advanced melanoma. JAMA. 2016;315:1600-9.

35. Capietto AH, Faccio R. Immune regulation of bone metastasis. Bonekey Rep. 2014;3:600.

36. Kawachi H, Tamiya M, Tamiya A, Ishii S, Hirano K, Matsumoto H, et al. Association between metastatic sites and first-line pembrolizumab treatment outcome for advanced non-small cell lung cancer with high PD-L1 expression: a retrospective multicenter cohort study. Invest New Drugs. 2020;38:211-8.

37. Tamiya M, Tamiya A, Inoue T, Kimura M, Kunimasa K, Nakahama K, et al. Metastatic site as a predictor of nivolumab efficacy in patients with advanced non-small cell lung cancer: a retrospective multicenter trial. PLoS One. 2018;13:e0192227.

38. Peters S, Cappuzzo F, Horn L, Paz-Ares L, Borghaei H, Barlesi F, et al. OA03.05 analysis of early survival in patients with advanced non-squamous NSCLC treated with nivolumab vs. socetaxel in CheckMate 057. J Thorac Oncol. 2017;12:S253. 